\title{
15 años de la Revista de Medicina y Cine
}

\author{
María GARCÍA MORO1, José Elías GARCÍA SÁNCHEZ2,3,4, Enrique GARCÍA SÁNCHEZ2,3,4, Enrique \\ GARCÍA MERINO5
}

\section{5 years of Journal of Medicine and Movies}

\begin{abstract}
${ }^{1}$ Facultad de Farmacia. Universidad de Salamanca (España). ${ }^{2}$ Departamento de Ciencias Biomédicas y del Diagnóstico. Facultad de Medicina. Universidad de Salamanca (España). ${ }^{3}$ IUCE (Instituto Universitario de Ciencias de la Educación). Universidad de Salamanca (España). ${ }^{4}$ IBSAL (Instituto de Investigación Biomédica de Salamanca) (España). ${ }^{5}$ Departamento de Sanidad, IES Martínez Uribarri, Salamanca (España).
\end{abstract}

Autor para correspondencia: José Elías García Sánchez. Correo electrónico: joegas@usal.es

Recibido el 24 de enero de 2019; aceptado el 30 de enero de 2019.

Cómo citar este artículo: García Moro M, García Sánchez JE, García Sánchez E, García Merino E. 15 años de la Revista de Medicina y Cine . Rev Med Cine [Internet] 2019;15(1):1-2.

DOI: https://dx.doi.org/10.14201/rmc.20244

La Revista de Medina y Cine / Journal of Medicine and Movies salió a la luz en enero del año 2005 por tanto, en 2019 se cumplirán los 15 años de su aparición. Esta efemérides queremos compartirla con todos vosotros, lectores y autores, que sois los verdaderos protagonistas ya que habéis hecho posible que este canal de conocimiento se haya convertido en una realidad.

La Revista de Medicina y Cine (RMC) se podría definir como una revista médico-humanista. Su objetivo fundamental es formar y despertar un espíritu crítico en los profesionales de las diferentes disciplinas relacionadas con la salud y en la población en general.

A lo largo de estos años, la RMC ha publicado numerosos artículos que relacionan el Cine con distintos aspectos de la Salud. Sería un arduo trabajo recopilar todos y cada uno de los ámbitos que se han abordado, pero baste como ejemplo citar algunos como: medicina, enfermería, logopedia, historia, enfermedades infecciosas, farmacología, microbiología, hematología, oncología, pediatría, psicología, psiquiatría, fisiología, nutrición, medicina preventiva, endocrinología, nefrología, neurología, dermatología, toxicología, traumatología, bioética, cuidados paliativos, medicina tradicional, ensayos clínicos, anatomía, cirugía, antropología, discapacidad, enfermedades raras, medicina de familia, experiencias docentes...1-13.
En estos años se han llevado a cabo dos estudios bibliométricos de la revista, el primero en el año 2009 (volumen 5, número 3) y el segundo en 2016 (volumen 12, número 2) que han ofrecido a los lectores un análisis detallado de la evolución de la RMC. Os invitamos a leerlos y a continuar esta interesante línea de trabajo14-15.

Con la experiencia adquirida con la RMC, se puede afirmar que el cine es un elemento complementario muy útil de la labor docente.

Es importante señalar que se han tenido que superar numerosas dificultades para consolidar la revista, tal vez la más relevante sea la falta de apoyo institucional de la Universidad de Salamanca, a pesar que el copyright pertenece a Ediciones Universidad de Salamanca, en todo lo concerniente al trabajo editorial y en lo relativo al posicionamiento en bases de datos internacionales. Una pena.

La RMC nació con la finalidad de ser una publicación que permitiera analizar los contenidos biosanitarios del cine con fines educativos, de mentalización y divulgación, dirigida a profesionales de ciencias de la salud, educación, comunicación y cine y a la población en general, pero con la idea de incorporar cualquier iniciativa, que fuera sostenible en el tiempo. De esta manera y teniendo en cuenta que una parte importante de los argumentos 
cinematográficos tienen su origen en la literatura, el Comité Editorial decidió dar cabida a una nueva experiencia, incluyendo, en su línea editorial, contenidos que relacionaran Literatura y Salud. En el año 2015 (volumen 11, número 1) se inicia esta nueva andadura. En una editorial de la Revista de Cine y Medicina, Guardiola y Baños se hacen eco de la inclusión de artículos no médicos en algunas de las revistas más relevantes de la medicina y enfatizan cómo las artes pueden contribuir a abordar un amplio espectro de aspectos relacionados con la medicina y la salud en general|16-17.

Otro aspecto relevante relacionado con la plasticidad que tiene la RMC es el comienzo de diversas colaboraciones en torno a la contribución de las obras de arte en la formación sanitaria. En el volumen 14 número 1 , se inició esta nueva andadura. Comienzan estas colaboraciones analizando una experiencia llevada a cabo en el colectivo de los estudiantes del Grado en Medicina donde se comentan diferentes aspectos de la relación que existe entre la medicina y el arte ${ }^{18}$.

En estos 15 años los auténticos protagonistas han sido los autores y sus artículos que han contribuido a descubrir la enorme producción cinematográfica que aborda temas de salud en sus diferentes ámbitos y a analizar las películas con una visión sanitaria. Todo esto ha quedado plasmado en análisis individuales o en comunicaciones de experiencias llevadas a cabo tanto en aspectos relacionados con la divulgación como con la educación. Comentarios similares se podrían hacer desde el año 2015 hasta la fecha en temas relacionados con la literatura o el arte como se ha comentado anteriormente.

Desde el principio se estableció que fuera una revista gratuita y en internet, algo no muy común en aquel entonces y además que cada artículo fuera revisado por pares.

A la hora de hacer un balance es importante destacar dos aspectos, por una parte la percepción de los usuarios sobre la calidad de la revista, que es muy buena y por otra el análisis de los fríos números que aportan cifras relevantes. A lo largo de todo su recorrido la RMC ha tenido más de 1.500 .000 de visitas de más de 100 países diferentes. En el último año se han descargado más de 215.000 artículos.

A todos vosotros, autores y lectores, os queremos agradecer vuestro esfuerzo y colaboración ya que habéis logrado que la RMC se haya consolidado y que podamos decir que es una realidad y, probablemente, un referente internacional en la utilización del cine en la docencia y divulgación sanitaria.

\section{Referencias}

1. Yagüe Renau A, Almudéver Campo L. La representación de la enfermería en el cine. Modelos y estereotipos. Rev Med Cine [Internet] 2018;14(3):165-71.

2. Carreras Panchón A. Albert Schweitzer (1875-1965): ficción y reconstrucción histórica en el cine. Rev Med Cine [Internet] 2013; 9(1): 31-42.

3. García Merino E, Bedoya Elena L, García Moro M, García Sánchez E, García Sánchez JE. Educando con el séptimo arte. La polio de Franklin Delano Roosevelt en el cine (III): Hyde Park on Hudson (2012). Rev Med Cine [Internet] 2018;14(4): 285-91.

4. Icart Isern MT, Díaz Membrives M, Icart Isern MC. Vida y muerte de un fármaco en el siglo XXI. Del libro Mediator ${ }^{\circledast} 150 \mathrm{mg}$. Combien de morts? (I. Franchon, 2010) a la película La fille de Brest (E. Bercort, 2016). Rev Med Cine [Internet] 2018;14(1): 5-12.

5. García Sánchez JE, García Sánchez E. Los padres de la microbiología en los carteles de cine. Rev Med Cine 2005;1 (2): 47-56

6. Domínguez Vías G. El Desastre del 96: Una forma educativa de explicar las reacciones fisiológicas producidas como consecuencia de la exposición a la baja presión de oxígeno a gran altitud usando la película Everest (2015). Rev Med Cine [Internet] 2018;14(4): 227-36.

7. García Moro M, García Sánchez E, García Sánchez JE. Traumatología y ortopedia: fractura espiral del radio en Dos buenos tipos (2016). Rev Med Cine [Internet] 2018;14(4): 293-96.

8. Cambra Badii I, Mastandrea PB, Paragis MP. El mandato del nacimiento. Cuestiones bioéticas y biopolíticas en la serie El cuento de la criada. Rev Med Cine [Internet] 2018;14(3): 181-91.

9. Pena Bandres I. Cuidados paliativos y cine: una actualización comentada de la producción relacionada desde mediados del siglo pasado hasta la última década del actual. Rev Med Cine [Internet] 2018;14(4): 259-65.

10. Moratal Ibanez L; Domínguez A, Wallinger M, Rubino P. Las medicinas tradicionales y los conocimientos ancestrales. El abrazo de la serpiente (2015). Rev Med Cine [Internet] 2018;14(3): 173-79.

11. Juanes Méndez JA. Despierto (2007): toda una pesadilla en el quirófano, durante un trasplante de corazón. 2015; 11(1): 5-12.

12. Badía Corbella M, Sánchez-Guijo Acevedo F. La representación de las personas con discapacidad visual en el cine. Rev Med Cine 2010; 6(2): 69-77.

13. Valero Marcet $M$, Pérez Sánchez J. Cine y práctica reflexiva. Una experiencia en los estudios de Medicina del Campus del Mar de Barcelona. Rev Med Cine [Internet] 2018;14(4): 253- 58.

14. Tarrés MC. Análisis bibliométrico de la Revista Medicina y Cine (2005-2008). Rev Med Cine 2009; 5(3): 106-15.

15. García Moro M. García Merino E, Tarrés MC. Analisis bibliometrico de la Revista de Medicina y Cine (2009-2014). Rev Med Cine [Internet] 2016;12(2): 91-7.

16. Baños JE, Guardiola E. Medicina y Literatura: el valor de las palabras. Rev Med Cine 2015; 11(1): 3-4

17. Fresnadillo Martínez MJ. Las enfermedades infecciosas en la literatura. Una larga historia sin final. Rev Med Cine 2015; 11(1): 41-53.

18. Hidalgo A, Hidalgo C, Cantabrana B. Contribución del arte a la formación de los estudiantes de medicina. Rev Med Cine [Internet] 2018;14(1): 53-62. 